\title{
System developments in the ACT language: Towards machine independence
}

\author{
J. R. MILLENSON \\ McGill University, Montreal, Quebec, Canada
}

\begin{abstract}
ACT (the Automated Contingency Translator) is a list-processing state-oriented sequence control language for on-line control and data acquisition of psychological experiments. Since 1967, ACT software has been progressively expanded and the machine base extended from the PDP 8 family to the PDP9, NOVA, and the PDP11. The most recent variant of this language, designated ACT-N, removes previous arbitrary restrictions on state network complexity, expands the conversational repertoire, and adds a subset of compatible BASIC to ACT, thereby giving the package greatly increased computational powers and data storage facilities.
\end{abstract}

The problem-oriented language known as ACT (Automated Contingency Translator) (Millenson, 1968, 1973) is one of a number (McLean, 1968; Polson, 1973; Snapper, Kadden, Knapp, \& Kushner, 1967) of sequence control languages for automating the experimental procedures of psychology. The first version of this lanaguage (ACT-I) (Millenson, 1967) was implemented on a $4 \mathrm{~K}, 12$-bit, PDP8/S computer, but because of the small memory, this version served only as a successful prototype for demonstrating the feasibility of the language. ACT-II shared the syntax and grammar of ACT-I, but was implemented on an 8K PDP8/S. A working system was in use for several years at the Oxford University Department of Experimental Psvchologv. time-sharing the control of four experiments, each limited to three digital response inputs, and nine digital stimulus outputs. Program input and output utilized Teletype consoles exclusively. ACT-III is a commercial variant of ACT-II marketed for PDP8 and Data General NOVA computers by BRS-Foringer (cf. Lehigh Valley Electronics, 1970), for use with their digital interface system known as INTERACT. Another version of ACT-II, called ACT-9, was implemented for the PDP9, and is currently in use at the Psychology Department, Birmingham University, England.

The present variant, ACT-N, written for both the NOVA family and the PDP11, is the most sophisticated version extant. ACT-N removes many of the arbitrary restrictions of previous versions, and greatly extends the computational powers grafted to the language. Thus, whereas other versions of ACT are limited to eight independent experimental stations, ACT-N is limited only by the number of available device codes for the processor. ACT-N provides for considerable storage of data in high speed core memory, increased calculational

My colleagues, Roger Loxton, Barry Litwin, and Gary Bernstein, have all made significant contributions to the continuing development of the ACT language described in this paper. The work has been supported in part by grants from the Science Research Council (United Kingdom) and the National Research Council of Canada. facilities including multiplication, division, and root extraction of real numbers, array manipulations, greater network complexity with infinite nesting and seven independent state systems (planes) per station, and multiconsole remote inputs. All of these improvements contribute to a language tailor-made to expedite the logic of experimental control and the retrieval and analysis of data. At the same time, ACT-N retains the simple English syntax which is so close to a natural language description of the procedure that a novice can learn and use it virtually immediately (Millenson, 1971).

\section{BEHAVIORIAL EXPERIMENTS}

The procedures of psychology are characterized by rules that are established by an experimenter between subject-generated events and experimenter-generated events. Subject-generated events are normally termed responses, although they may be, and often are, emitted freely without any observed elicitor. Since the experimenter-generated events are presented in the environment of the subject, they are normally termed stimuli. The rules established between responses and stimuli are technically known as contingencies, and they may be determinate or probabilistic.

Typical examples of responses are pressing keys and pedals, turning wheels and pushing panels. Typical examples of stimuli are the delivery to subjects of objects, rewards, patterns of lights, tones, shocks, at various angles, orientations, intensities, and frequencies. The stimuli and responses that ACT deals with are those that can be digitalized, that is, specified in terms of their occurrence or nonoccurrence. Automating the contingencies of a psychological experiment is desirable whenever accurate measurement of time intervals is necessary, the contingency network is complex or repetitive, the set of possible ditterent responses and stimuli that must be treated is large, or when the procedure itself depends on the outcome of calculations made on immediately preceding results. Except for the latter condition, a digital computer is rarely essential for 
the automation process. However, when numerous experiments are replicated frequently and whenever great flexibility in modifying experiments is required, the cost of hard-wired circuitry begins to approach that of a general purpose computer which will do the same job, and others as well.

Nevertheless, acquiring a digital computer is only the first, and often easiest step, in achieving a computer control system for experimentation. Two other crucial steps consist in writing the desired programs to implement the experimental logic, and constructing the necessary circuitry to bridge the interface between an actual experimental environment and the computer itself. These two problems are known in computerese as the software and hardware problems, respectively, and solving them is what ACT and other similar problem-oriented languages are designed to do.

\section{INTERFACE HARDWARE}

In ACT-N, up to 15 digital responses (R) events from, and 15 stimulus (S) events to, an experimental subject appear at a device known as a station (Figure 1). Each bit is assigned an octal value from 1 to 40,000 (40K) which also serves as its internal representation. Stimulus and response bits are fully buffered, with 1-A reed relay contact closures serving as stimulus output devices, and switch closures to ground accepted as response inputs.

Although it is frequently possible, where only a few stimulus and response bits are being used, to run several experiments from one station, it is conceptually simpler for the purposes of exposition to think of one station corresponding to one experiment. Thus when many experiments are being automated concurrently, the response and stimulus bit status of many such stations as shown in Figure 1 will be independently sensed and set, respectively. The speed of minicomputers is such that this task is completed in a few milliseconds, and the appearance of simultaneous control is therefore given, even though the control is actually time-shared.

Although it is rare for any one experiment to use as many as 15 independent digital responses, it does occasionally happen that more than 15 unique stimuli are required. Two stations could be combined to double the available stimuli, but this is uneconomical. Another method, used when many of the potential stimulus possibilities are mutually exclusive, is to decode some of the stimulus bits. Thus if six bits are decoded, leaving

\begin{tabular}{|c|c|c|c|c|}
\hline & & [STATाOH S & & \\
\hline ○ ○ ○ & () $\odot$ & (a) & ○ ๑ $\odot$ & $\left(\circ \odot_{\mathbf{s}}\right.$ \\
\hline 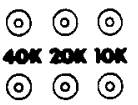 & 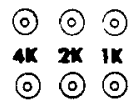 & 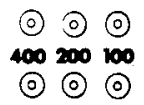 & 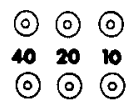 & 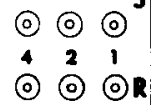 \\
\hline
\end{tabular}

Figure 1. 15-bit parallel station interface for response (R-input) events and stimulus (S-output) events.
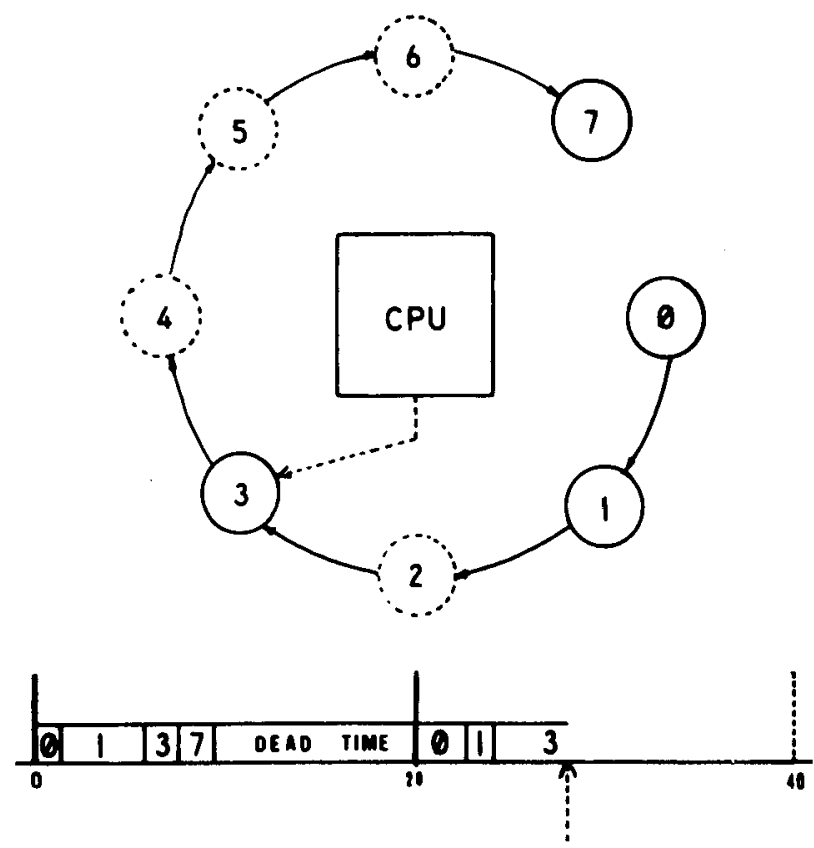

Figure 2. Round Robin service of ACT stations by central processor.

the remaining nine to work independently, $2^{6}$, or 64 , mutually exclusive stimuli are available in lieu of the six independent and overlapping stimuli of the original six output lines.

The operation of the station hardware in an active station is as follows. On program command, a binary number corresponding to a stimulus configuration is strobed from the central processor to the station stimulus bits. Since the stimulus bits correspond to flip-flop circuitry, these values remain on the reed relay bank until and unless their associated program changes them. Whenever a running program is terminated, however, all stimulus bits are automatically turned off. Response bits also correspond to flip-flops. They are reset to off (zero) at the initiation of an ACT program for the appropriate station, and set (one) only by the leading edges of inputs (switch closures) from the subject. The response bits are examined periodically, being reset immediately after being examined. Since the offset of a response is not known to the interface, on those occasions when this information is needed (e.g., response duration studies) it must be converted to a leading edge and input to a different response bit.

Two additional items of hardware complement the stations. The first of these is an adjustable clock that determines the frequency of updating each station. The ACT-N clock is a crystal stabilized multivibrator which interrupts the central processor at the desired frequency. Sixty Hertz is a typical value which permits the time-sharing of 20 or more stations. The clock can run 10 to 20 times faster giving time resolutions down to $1.2 \mathrm{msec}$, if only one or two stations are running, so that special work on short reaction times or neuroelectric 
potentials is feasible, though expensive.

ACT serves its stations in a round robin fashion as illustrated in Figure 2. Two clock cycles are shown and four stations $(0,1,3,7)$ out of an eight-station configuration are running, with Station 0 presumed to be first in the queue. The amount of time allotted to any station on any given tick is variable, since that time will depend on what is to be done to update the particular experiment. At the data rates of most behavioral experiments, most of the time between cycles is dead time; nonetheless, if data are not to be lost, such a real-time system must be built around the rare worst possible case when all stations are active and all have maximum overhead. Dead time is, of course, far from wasted time. It is used for background assembly of incoming programs, computation, output and, although not yet implemented, certainly available for disk or magnetic tape storage and retrieval of data.

The second special hardware device used by ACT is a seven-bit random number generator which allows random selection of intervals, and haphazard presentation of stimulus events (Millenson \& Sullivan, 1969). The peaks of seven noisy diodes are used to independently toggle seven flip-flops which are referenced as a parallel word every tick of the ACT clock. The method generates independent words at speeds up to $2 \mathrm{KHz}$ or better, thereby eliminating the need for built-in tables of random numbers which occupy valuable memory space, are tedious to construct, and in any case are only approximately random.

\section{STATE DIAGRAMS}

The contingencies of behavioral experiments may be specified as a finite set of states, and transitions between states. Examples of states are the appearance in a tachistoscope of a nonsense syllable to a subject with the proviso that if he says "bik" we shall call it a correct identification; the delivery of a $1 \mathrm{sec}$ electric shock to a rat's grid floor; the condition such that if a monkey presses a panel 42 times food will be presented. A state is clearly a particular configuration of the subject's environment lasting for a specified duration, and combined with a set of potential changes that may occur if the subject behaves in prescribed ways.

The events which lead to transitions between states are fortunately restricted to only three basic types. Some examples are a pigeon's key peck which turns off a center disk light and illuminates two side key lights; the completion of a time interval that a signal is allowed to be present; the termination of an experiment after 100 reinforcements. In every case, one state is terminated and a new state begun. These three transition examples may be described more generally as: (1) the occurrence of subject-initiated response events; (2) the sheer passage of time; and, (3) the occurrence or repetition of a state itself, a kind of falling domino transition.
Because of the conceptual economy in state description, ACT represents psychological or behavioral experiments as a network of states and transitions. In the three-state diagram of Figure 3, states are represented by rectangles, and transitions are vectored lines connecting them. The diagram illustrates the operant reinforcement schedule known as differential reinforcement of low rates (DRL). The diagram is read from left to right, and for no other reason than that they were drawn in that order, the states are labeled S1, S2, and S3, where S standards for "state." Underneath each state label is a second number, the octal number corresponding to the stimulus bits that ACT-N is to actuate at the station inferface as long as that particular state is in effect. The procedure begins, by convention, at the leftmost state, S1, and the unattached arrow at the left implies that $\mathrm{S} 1$ is automatically initiated by program commencement. In $\mathrm{S} 1$, a response event at Bit 1 (symbolized as $\mathrm{Rl}$ ) anytime during that state reinitiates that state which, following a rule that says that entry to a state always resets all its transition qualifiers, means that the 30 -sec transition is reset. If, however, 30 sec elapse without an interruption from R1, then the network passes directly to $S 2$. But since $S 2$ is still associated with Stimulus Bit 1, the transition is not distinguished by a stimulus change in the subject's environment. Nevertheless, the conditions of the experiment have changed ( $\mathrm{R} 1$ will now lead to Stimulus Bit 4), so a new state (S2) is needed to reflect that fact. The experiment will rest at S2, Stimulus 1, until and unless R1 occurs, at which time it will move to S3, and produce Stimulus 4 for $2 \mathrm{sec}$, after which the procedure recycles. If we connect Stimulus Bit 1 to a houselight, Stimulus Bit 4 to a solenoid that raises a food tray, and Response Bit 1 to a lever, we have, in fact, exactly described DRL 30-sec for lever pressing with $2 \mathrm{sec}$ reinforcement. And, surprisingly enough, once we have the computer ACTified, getting from the diagram to the actual experiment is just that easy.

integer variable: A single computer register holding a positive integer from 0 to 65,535 , and symbolized as a letter from A through $\mathrm{N}$ inclusive. Thus, a somewhat more sophisticated DRL procedure is the one Figure 4, where absolute values of stimuli, responses, and time are replaced by the appropriate variable values $\mathrm{A}, \mathrm{B}, \mathrm{C}, \mathrm{J}, \mathrm{K}$, $\mathrm{M}$, and $\mathrm{N}$. If $\mathrm{K}$ happened to be set to $1, \mathrm{M}$ to $30, \mathrm{~J}$ to 1 ,

One ostensible advantage in computer control over other forms of automation is its greater flexibility for changing and modifying procedures, a familiar characteristic of that trial and error method we dignify

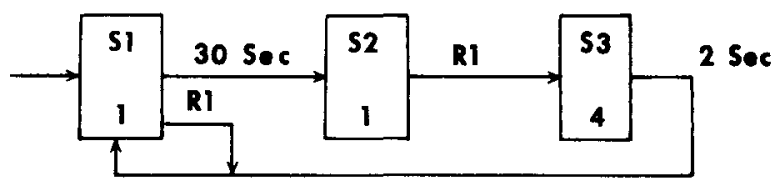

Figure 3. DRL 30-sec state fragment. 


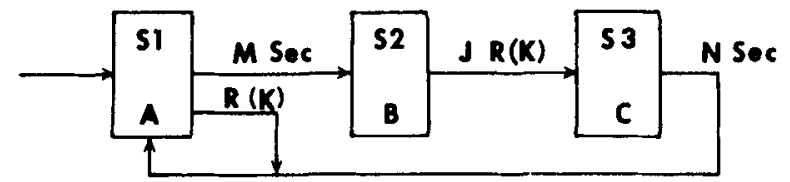

Figure 3 . Generalized DRL state fragment.

as scientific research. The diagram of Figure 3 is notable in that the value of all stimuli, responses, and time intervals are fixed. Nevertheless, in ACT-N they can all be variable. The simplest kind of variable in ACT $-\mathrm{N}$ is an A to $1, B$ to $1, \mathrm{C}$ to 4 , and $\mathrm{N}$ to 2 , Figure 4 would produce precisely the procedure of Figure 3 . On the other hand, Figure 4 is capable, merely by setting the values of its parameters before, or changing them during, the experiment of producing an entire family of DRL procedures.

Figure 5 complicates still more the basic DRL paradigm to illustrate the nesting of state networks. Suppose we desire the experiment to end after either 50 reinforcements $(50 \mathrm{~S} 3 \mathrm{~s})$ or $1 \mathrm{~h}$, whichever occurs first. Figure 5 produces that effect by creating two new states, $\mathrm{S} 4$ and S5. Additionally, the diagram illustrates a variable, $K$, being counted up in $S 3$, and being detected as the transition for superstate $\mathrm{S} 4$ to a terminal state (one lacking transitions) S5. The notation at the top of the large rectangle that represents $S 4$ indicates that the variable $K$ is zeroed at the initiation of $S 4$, and the notation above $\mathrm{S} 3$ indicates that $\mathrm{K}$ is incremented each time that $\mathbf{S} 3$ is produced. In general, associated with any state may be a block of computation in the language of BASIC which is carried out the moment the state is entered, and not again until the state is reentered. ACT and BASIC share the integer variables and communicate through them. Thus, the values of the integer variables $A$ through $\mathrm{N}$ are available to transition statements which can sense their values to determine whether or not conditions have been met for changing states.

The nesting of states shown in Figure 5 occurs in many procedures. Often an overall main procedure is in effect, and subprocedures are nested within the overall procedure. Partition of experimental sessions into different periods for selective counting and recording of data is expedited by nesting. In ACT-N, nesting can be indefinitely deep, and the value of the stimulus delivered at the interface station is the logical product of the stimuli assigned to all the currently active states in the nest.

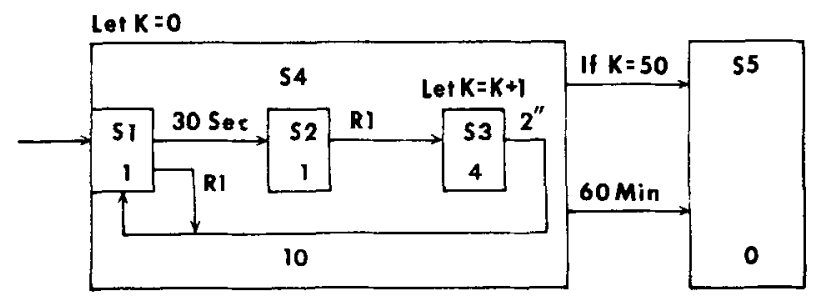

Figure 5. DRL nested.
Experiments very often involve independent timing cycles or independent procedural fragments that overlap one another. Suppose, for instance, that we desire to superimpose a Pavlovian conditioned emotional response (CER) procedure over the instrumental DRL schedule of Figure 5. One simple way to accomplish this is simply to define a second state fragment, or state plane, that starts out with the first one and operates completely independently of it (Figure 6). The use of probabilistic time transitions in the lower system of Figure 6 provides intertrial intervals (ITIs) of 8-min average duration with a 1 -min minimum, CS durations averaging $60 \mathrm{sec}$ with a minimum value of $15 \mathrm{sec}$, and a UCS of $1-\mathrm{sec}$ duration. $S 1$ in the lower system commences simultaneously with $\mathrm{S} 4$ and $\mathrm{S} 1$ in the upper system (the dotted vertical line suggests this synchrony by "ganging" the two systems). Parallel state planes permit the user to fractionate station interfaces and use

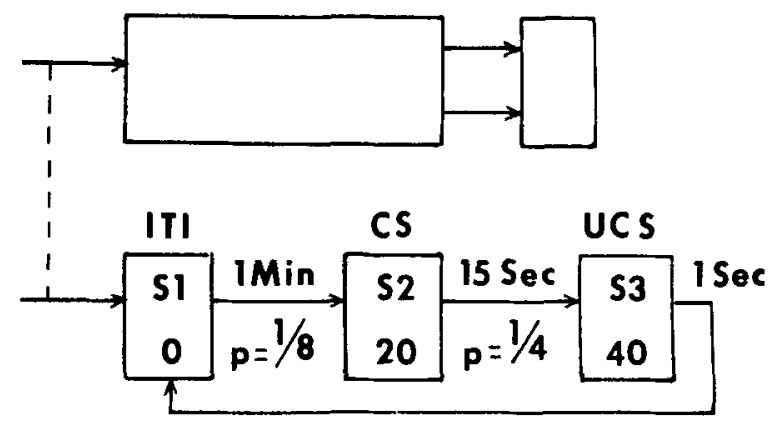

Figure 6. CER superimposed over DRL of Figure 5. See Figure 5 for details of the upper state system which is shown here only schematically.

the same station for more than one experiment. In principle, seven simple experiments could be run from one station, if no more than two or three stimuli and responses were needed for each, and if no independent timing cycles or parallel networks were needed in any experiment.

\section{ACT-N SYNTAX}

One day, perhaps, computers will be able to read state diagrams directly. However, at the present time, input to minicomputers is typically by coded alphanumeric symbols entered from punched paper tap or Teletype keyboard. Consequently, a meta-language, the ACT "language," in fact, was evolved to bridge the gap between pictorial state diagrams and the internal data structure of a computer that corresponds to such diagrams. The syntax of this meta-language has been described elsewhere (Millenson, 1968, 1971, 1973) and, because ACT-N differs only minimally from earlier dialects, will not be treated in detail here. In brief, it consists of a simple set of English statements which are combined according to a fixed set of rules to correspond point for point with the properties of a state diagram. These statements are then entered from the Teletype 
console and assigned to a given idle station, under control of a one-pass assembler. At any given time, each station may have at most one such set of statements associated with it, called its ACT-N program. A station with a running program is said to be active, and its variables may be modified through interactive conversation. (The complete program for the DRL-CER experiment of Figures 5-6 is shown in Appendix A).

\section{BASIC COMPUTATIONS ON ACT VARIABLES}

Amalgamated with the ACT language for specifying the logic of procedures and operating on its variables, is a subset of the arithmetic procedure oriented computer language known as BASIC (Farina, 1970; Hare, 1970). The combination of these two different, but powerful, dialects, each with its own domain of applications, identifies ACT-N not only as a computer language, but also as a sophisticated process control system. In that system, BASIC instructions are written in a separate assembly phase that follows directly on from the end of the ACT procedure phase of assembly. The BASIC commands implemented for use with ACT-N are:

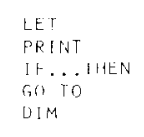

In ACT-N, a set or block of BASIC instructions may be associated with any of the states previously declared in the ACT procedure section. There is no limit as to the number of BASIC statements in a block; however, only seven statement labels are available. Nevertheless, since only lines actually requiring labels for transfer of control need be numbered, the number of permissable statements per block will generally not be a constraining factor.

Each block of BASIC statements may be thought of as a miniature BASIC program, complete in itself. Although reference may be made to common variables, there is no way that control can be transferred across blocks associated with different states. Execution of BASIC blocks takes place as soon as possible after entry to their associated states. And each time the associated state is entered the BASIC block will be executed again.

The time taken to execute the block will, of course, depend on the number and type of instructions to be treated and the presence or absence of loops. The processor is designed so that blocks containing any time-consuming routines such as multiplication $(385 \mu \mathrm{sec})$, division $(700 \mu \mathrm{sec})$, square root extraction (maximum of $7 \mathrm{msec}$ ), and printing (100 msec per character) are queued up in background and gradually executed as time permits in the dead time of Figure 2. However, if none of these routines is called, the block will get executed in foreground along with transition testing, response sensing, stimulus generation, and similar real-time chores. Therefore, it is the responsibility of the programmer to ensure that lengthy loops cannot occur in foreground tying up the ACT processor for significant portions of the clock cycle, thereby preempting normal processing of other running stations. In the worst possible case where a loop happens to take longer than the clock cycle itself, the offending program will be automatically killed, and an error diagnostic sent so that other users will not suffer.

BASIC blocks to be executed in background are placed on a first-in-first-out list which is gradually processed as dead time allows. At present this list is $57_{8}$ long and, if an attempt is made to queue more than 57 such blocks, an error diagnostic will be delivered and the excessive block will not be executed at all. The background BASIC interpreter processes these blocks one at a time in the order they are received. Since some blocks may have lengthy printing sequences on slow hardcopy devices, the time taken to execute completely a background BASIC block, measured from entry to its associated state, is indeterminate. If the queue is short and no printing is involved, the block could get executed within a few dozen milliseconds of state entry. On the other hand, if 56 blocks are queued ahead of it, some of them requiring output taking 10 to $15 \mathrm{sec}$, it could be several minutes before a block is executed. For this reason the programmer must carefully assess the user environment in which he is an element when performing background calculations, the results of which will be required to update variables that play a critical part in the real-time procedural flow.

In the BASIC section of ACT-N, the user must specify to the ACT assembler the particular state to which the following BASIC statements apply. Thereafter he writes the BASIC statements one to a line, terminating the block with an extra carriage return/line-feed sequence. A typical block of BASIC is the following:

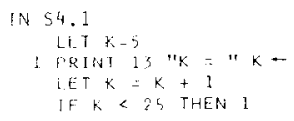

This little block of BASIC causes the values of $\mathrm{K}$ from 5 to 25 to be printed out on output device 13 (high-speed punch). Because it contains a PRINT instruction, the entire block will be treated in background.

In ACT-N there are four distinct classes of variables: (1) the integer variables, A, B, C, . N discussed above in several contexts, and used principally for counting, simple addition and subtraction, and for logical operations; (2) 127 special integer variables, Q1 to Q127 for recording data (see below); (3) fixed decimal point "real" variables with fractional components, W, X, Y, and $Z$, used principally for multiplication, division, root extraction, and complex function generation; (4) array integer variables, $U$ and $V$, used principally to store tables, lists, and incoming data, and to collect frequencies for histograms. 


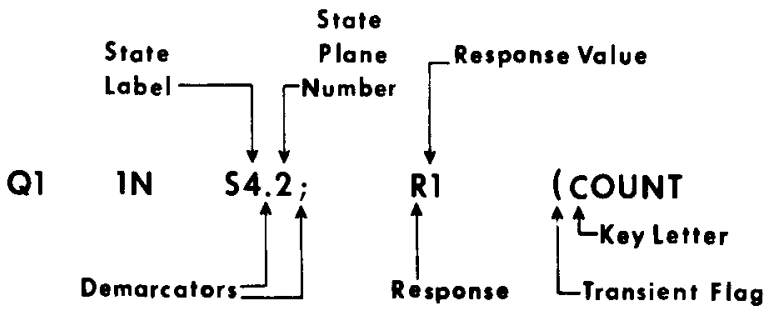

Figure 7. A generalized record statement.

By use of the LET statement, the BASIC variables may be assigned values, added, and subtracted to one another; and in the case of the reals, divided, multiplied, and raised to the power of $1 / 2$ (SQRT), as well. The IF ... THEN statement makes comparisons and transfers of program flow within a BASIC block. The total effect of the BASIC package within ACT-N is to produce a system capable of quite sophisticated on-line computations whose outcomes can enter into the real-time state networks to generate time intervals, stimulus values, and contingency decisions based on very complex preassigned or interactive functions.

The purpose of the PRINT command is to output for permanent storage, experimental data, the results of computations, and special messages. Because ACT-N is designed to work with a number of different hardcopy devices interfaced to the central processor, it is necessary to specify in each PRINT statement exactly which output device is to receive the data. It is also necessary, since different users may be calling the same device closely in time to have more intimate control over the formatting, especially in controlling the return of the carriage and new lines, than is customary in ordinary BASIC implementations.

The general format of the PRINT statement is:

PRINT 11 - "TEXY" A, B, W, X, U(10), V(9), U(J), Q12 "MORE TEXT"-

The octal number after the PRINT command specifies the output device, in this case a model ASR33 Teletype Punch and Printer operating synchronously at 10 characters per second. A carriage return/line-feed character $(\leftrightarrow)$ appears next followed by a portion of text, enclosed in quotation marks. All the ASCI printing characters from space (040) through $\uparrow(136)$ are available. Although unlike general BASIC, expressions are not permitted within a PRINT statement, all the legal variables (integers, reals, arrays, and record) may be printed directly merely by naming them outside of quotations. The example ends with more text and a final $\leftarrow$ which causes another new line to be produced. The BASIC TAB function for spacing and page formatting is also available.

\section{RECORDING THE DATA}

The raw data of a psychological experiment that is concerned with digitalized behavioral events consists of response and stimulus counts of various types, taken at various times and places in the procedure, and the measurement of time intervals. All other data of interest can usually be synthesized from these raw elements.

Collecting the raw counts and durations is the function of a special set of 127 integer variables Q1, Q2, ... Q127. These variables correspond to line numbers typed by the assembler in a separate "record" phase. Two classes of data can go into a $Q$ variable. The first and simplest class refers to counts or durations of states. The form of these record statements is illustrated by the statement

$$
\text { Q1: } \$ 44.6 \text { COUNT }
$$

The assembler types the $Q$ line number. The programmer types the state (S44) about which information is desired, a point, and then the state plane (6) in which this state is located. The final word indicates what data to collect in Q1. "COUNT" in this statement refers to the number of times that the particular state in question was entered. If the duration that a state lasts is of interest, then the words UNITS, SEC, or MIN could have replaced COUNT in the example above. The durations of states may be cumulated over all repeated entries to the state, or the times may be specified as transient durations measured from the last entry. To distinguish the type of time value collected, the programmer encloses the same time units in parentheses, as in the example

$$
\text { Q17: } 544.6(5 E C)
$$

to obtain transient durations.

A second class of record statements is concerned with collecting response counts. Since response counts are generally desired in particular states the form of the statement follows the syntax shown in Figure 7, where the meaning should be self-evident. The left parenthesis before COUNT implies, as it did in recording times, that a transient count is wanted which will be reset to zero every time S4 in Plane 2 is entered. If a cumulative count is desired, the parenthesis are omitted.

Typically raw data are held in $Q$ variables for only a short time. This is because data are generally collected repetitively, and so old values are moved from volatile $Q$ variables into semipermanent places like arrays, perhaps after a modicum of analysis, making room for the next collection of another instance of the same datum.

\section{ACT-N SECTIONS: A FUNCTIONAL ANALYSIS}

ACT-N software consists of an assembler, an interpreter, and a monitor which determines which of these two subprograms shall be allowed to operate at 
any moment of time. Monitor also converses with the users via Teletype consoles and is responsible for returning the space used by dead programs to an available space list, giving out program headings and special messages, and producing the error diagnostics that occur during the running of a program. A simplified block diagram of the relations between the various components of ACT-N appears in Figure 8, divided into background (top) and foreground (bottom) activities.

Whereas the assembler operates only in background, portions of the interpreter operate both in foreground and in background. Foreground is simply the processing that is done to the running stations whenever the clock ticks: reading in of response and probability buffers, testing of transitions, effecting transitions, issuing stimuli, recording data in $Q$ registers, performing short BASIC routines associated with newly entered states, stacking pointers to longer BASIC sequences, and stacking the pointers to any programs that have just been killed. When all that is done, monitor moves the processing to background where incoming programs are assembled, long and slow BASIC blocks are executed, program variables are modified in conversational-interactive mode, and the garbage collector returns blocks from killed programs to available space.

Monitor normally rests in a quiescent mode, when none of its sections (the upper four rectangles of Figure 8) have any work to do, and in which conversation section recognizes only one character from either Teletype, control-E. To assemble an ACT-N program, the control-E key is struck on a Teletype console, causing the conversation section to respond either with WAIT if another user is already in conversation on another console, TRY LATER if another user is assembling a program from another console, or

$$
\text { STATION: }
$$

In the latter case, the user may now type an octal number corresponding to any one of his existing station

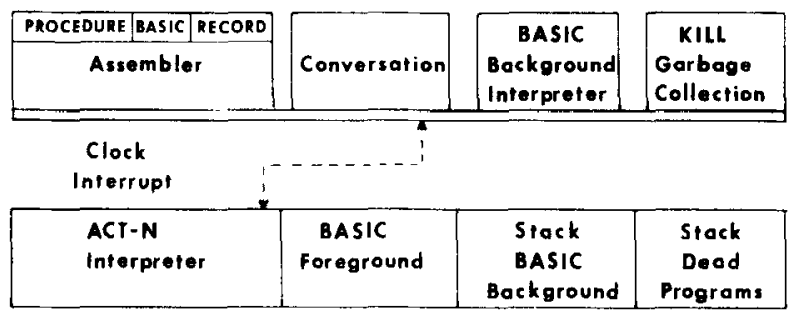

Figure 8. Functional block diagram of ACT-N principal components. Monitor has four background sections (top) that it services in a Round Robin, allocating a certain portion of background time to each. When the clock ticks, monitor interrupts this servicing, and control goes to the foreground interpreter section (below) which executes the four foreground sections in order for each acive station.
Table 1

Conversational Commands and Their Effects

$\begin{array}{ll}\text { Command } & \text { Effect } \\ \text { Control } F & \begin{array}{l}\text { Restart the program to its initial states } \\ \text { and zero all Q variables. } \\ \text { Kill the existing program in this station. }\end{array} \\ \text { Control V } & \begin{array}{l}\text { Causes the value of the integer variable } \\ \text { (K) or tixed element }(9) \text { of array (U) } \\ \text { to be typed }\end{array} \\ \mathrm{U}(9) \text { Carriage return } & \begin{array}{l}\text { Assigns the value 500, to } \mathrm{K} \text { or } \mathrm{U}(9) \\ \mathrm{K}=500 \mathrm{U}(9)=500\end{array} \\ \mathrm{~V}=15,2, \emptyset, 1234,8 & \begin{array}{l}\text { Sets } \mathrm{V}(1) \text { to } \mathrm{V}(5) \text { with this list of } \\ \text { decimal integers }\end{array}\end{array}$

device codes followed by a carriage return. If that station is free, conversation mode responds with the approximate number of lines available for programming (actually the number of eight-word core blocks free for program storage) followed by the word IN: ACT-N is now in assembly mode, procedure phase, waiting for the statements of a program which will be accepted from any of several peripheral input devices.

During the procedure phase of assembly, the state network is described in ACT syntax. When the " $\$$ " terminator of the procedure phase is received, the assembler moves to the BASIC phase, and now accepts statements in the BASIC language. A "\$" character also terminates the BASIC phase, and the assembler advances, after typing the word RECORD, to the record phase of the assembly, where the $Q$ variables, if any, are now specified by the user. A third and final " $\$$ " terminates this last phase, enters the assembled program into the list of running stations, and produces a message of the form

$$
14: 21 \text { PROGRAM IN }
$$

Monitor then returns the keyboard to quiescence.

\section{CONVERSATION MODE}

If the station number typed after control-E STATION: refers to an already running station, ACT-N responds with "[]" and remains in conversational mode. In this mode, the user may interact with an existing program to kill it, reset it to its initial states, or interrogate or modify its variables. In order to prevent a remote user from tying up conversation mode indefinitely, this mode waits a maximum of $35 \mathrm{sec}$ for a character, after which, failing to receive one, it terminates itself. Table 1 lists examples of the legal conversational commands and their effects.

\section{THE ARCHITECTURE OF ACT-N}

The ACT family may well be unique in sequential control languages for psychology in its use of an internal list structure for representing states, transitions, BASIC statements, and even variables. In a list structure, core 


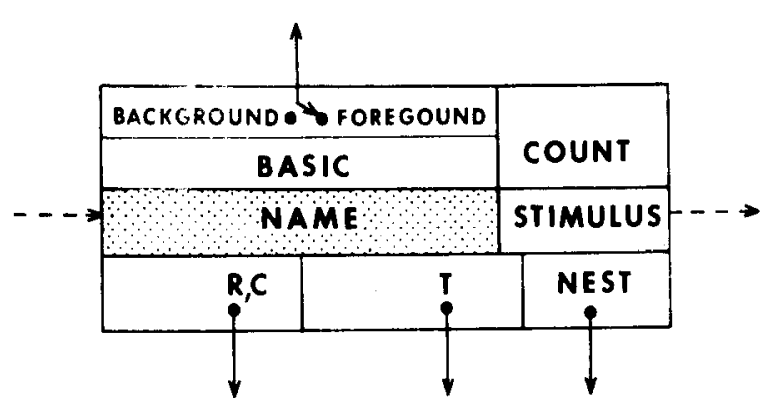

Figure 9. Core representation of a generalized ACT-N state.

memory is ordered associatively rather than sequentially (Forster, 1967; Millenson, 1970) with units of memory scattered haphazardly throughout available core. The unit of memory used by ACT is a "block" of eight sequential computer words, with the eighth word always containing the address of (link to) the next block on the list. All memory not utilized for the systems software itself is initialized into a list called the available space list. In $8 \mathrm{~K}$ NOVA configurations, the initialized available space list provides 332 blocks. For each additional $4 \mathrm{~K}$ of memory, an additional 4096/8 $=512$ blocks become available. Moderately complex experiments average about 75-100 blocks, so that to run a dozen experiments comfortably requires a $16 \mathrm{~K}$ machine.

The great advantage of a data structure in list format lies in flexibility and memory protection. The former is a consequence of being able to extend a list to any desired length, merely by tacking another element (usually a single block) to it. Thus, there is no limit to the number of states in a program, because states are eight-word blocks of memory linked together on a state list. Each state has a list of transitions (the transition list pointer is one of the eight words of the particular state block) which itself can be indefinitely long. An example of the core representation of an ACT-N state is shown in Figure 9. The eight words available correspond (1) to a pointer to the BASIC code, if any, associated with this state, and a flag to indicate whether it should be executed in foreground or background; (2) the $Q$ register for counting the number of entries to this state; (3) the internal name of this state; (4) the bit configuration (stimulus) to be issued when this state is active; (5) a pointer to any response (R) or integer variable comparison (C) transitions; (6) a pointer to any time (T) transition; (7) a pointer to any state currently nested below this state; and (8) the link to the next state in the list of states that defines the complete program for this station.

Programs themselves form a list of running stations to which members can be deleted or added at any time, and this list holds blocks with the information shown in Figure 10.

Memory protection is important when a number of independent users are sharing the same central processor.

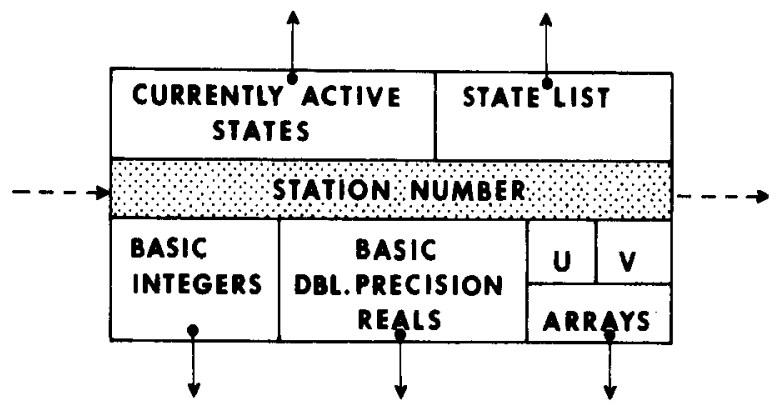

Figure 10. Core representation of the "headblock" of each running station. These are linked together to form a list of running stations.

Because of the way that memory is allocated and because ACT-N programs are interpreted rather than executed as compiled code, it is simply not possible for price must be paid for these conveniences; because blocks do not contain compiled code, but rather contain information which is interpreted by a master program, processing of this structure is slow, relative to a program of compiled instructions. Coursing up and down lists representing several dozen active states per any one user's program to affect any other user's. A time-consuming job. Indeed, the earliest implementation of ACT on a $16 \mu \mathrm{sec}$ cycle machine (PDP8/S) found four stations to be the unper limit that could be serviced in a clock cycle of $50 \mathrm{msec}$. Third generation minicomputers, however, solve the speed problem by brute force. The NOVA 800 on which ACT-N is implemented at Oxford University and the PDP11/10 at McGill are approximately 15-20 times faster than the PDP8/S, and these machines run several dozen stations quite comfortably with $16-20 \mathrm{msec}$ clock resolution cycles.

Another problem, not so easily solved, is that of optimal data structure incompatibility between ACT and BASIC. The computations of BASIC and the representation of arrays and variables, are poorly suited to an associative list structure. For instance, in ACT-N, to assemble an array 127 long, 16 consecutively located blocks must be found. As long as there are a few hundred blocks available when an array that size is requested, then no problem occurs; but when blocks are scarce, it might be impossible to find that much consecutive core, even though far more than 128 registers are available strewn about memory.

Thus, like most technical innovations, the current working design of ACT-N remains a compromise between speed and flexibility, reliability and parsimonious use of memory, generality and power. As yet, no systematic comparison of the various control languages in psychology has been carried out, so it is :00 early to assess the individual features of this system in the wider context of computer control. As for its immediate uses, the test of a language is in what can be said easily and parsimoniously in it, and for that we wait the judgment of the users. ${ }^{1}$ 


\section{APPENDIX A}

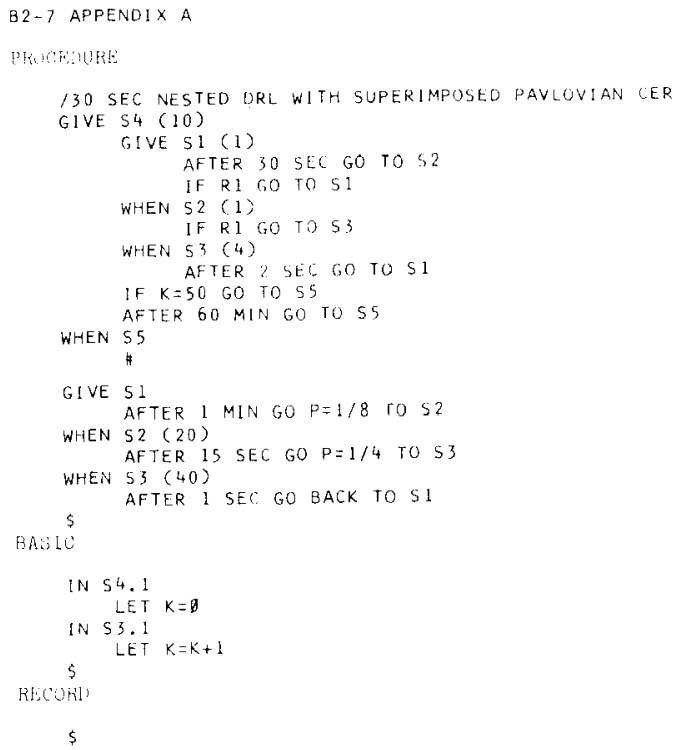

\section{REFERENCES}

Farina, M. V. Elementary BASIC with applications. Englewood Cliffs, N.J: Prentice-Hall, 1970.

Forster, J. M. List processing. London: Mac Donald, 1967.

Hare, V. C., Jr. Introduction to programming: A BASIC approach. New York: Harcourt, 1970.

Lehigh Valley Electronics. The ACT-III primer: Computer language and hardware interface for controlling psychological experiments. Lehigh Valley Electronics, Fogelsville, Penna, 1970.

McLean, R. S. PSYCHOL: A formal language for the control of psychological experiments. Unpublished Ph.D. thesis, Carnegie-Mellon University, 1968.

Millenson, J. R. An automatic contingency translator (ACT-I): A computer system for process control of psychological experimentation (Abstract). Psychonomic Bulletin, 1967, 1, 17.

Millenson, J. R. A general language for on-line control of psychological experimentation. Proceedings of the DECUS Society, April 1968, 137-142.

Millenson, J. R. Language and list structure of a compiler for experimental control. Computer Joumal, 1970, 13, 340-343.

Millenson, $J$. R. A programming language for on-line control of psychological experiments. Behavioral Science, 1971, 16 , 248-256.

Millenson, J. R. On-line sequential control of experiments by an automated contingency translator. In B. Weiss (Ed.) Digital computers in the behavioral laboratory. New York: Appleton-Century -Crofts, 1973. Pp. 1-39.

Millenson, J. R., \& Sullivan, G. D. A hardware random number generator for use with computer control of probabilistic contingencies. Behavior Research \& Instrumentation, 1969,1, 194-196.

Polson, P. G. SCAT: Design criteria and software. Behavior Research Methods \& Instrumentation, 1973, 5, 241-244.

Snapper, A. G., Kadden, R. M., Knapp, J. Z., \& Kushner, H. K. A notation system and computer program for behavioral experiments. DECUS Biomedical proceedings, June 1967

\section{NOTE}

1. ACT-N software for NOVA and PDP11 systems is available from BRS-Foringer Co., 5301 Holland Drive, Beltsville, Maryland; or Campden Instruments Ltd., 186 Campden Hill Road, London, W. 14, England. 